\title{
Surgical Outcomes of Minimally Invasive Transforaminal Lumbar Interbody Fusion for the Treatment of Spondylolisthesis and Degenerative Segmental Instability
}

\author{
Yung Park, Joong Won Ha, Yun Tae Lee, Hyun Chul Oh, Ju Hyung Yoo, Hyung Bok Kim \\ Department of Orthopedic Surgery, National Health Insurance Corporation Ilsan Hospital, Goyang, Korea
}

\begin{abstract}
Study Design: This is a retrospective case study.
Purpose: This study was designed to analyze the surgical outcomes of patients who underwent minimally invasive transforaminal lumbar interbody fusion (TLIF) for the treatment of spondylolisthesis and degenerative segmental instability.

Overview of Literature: If the surgical outcomes of a procedure are evaluated together with multiple indications, it is not clear how the procedure helped each subgroup of patients. For the reason that some indications achieve better outcomes than the others, we performed a subgroup analysis using validated outcome measures to demonstrate the optimal indications and the treatment results of TLIF.

Methods: We conducted subgroup analyses by comparing the prospectively collecting data from the consecutive patients who underwent single-level minimally invasive TLIF for the treatment of the following 3 subgroups of indications: 23 cases of low-grade spondylolytic spondylolisthesis, 24 cases of degenerative spondylolisthesis, and 19 cases of degenerative segmental instability.

Results: The average duration of follow up was $36.1 \pm 9.9$ months (range, 24 to 63 months). The preoperative pain and dis ability scores were significantly improved at final postoperative follow-up in all the subgroups (all measurements: $p<$ 0.0001). The 3 subgroups exhibited an equivalent improvement of the pain and disability scores at the final follow-up. The rates of radiographic solid fusion and complications were also similar among the 3 groups.

Conclusions: Our data suggests that minimally invasive TLIF optimally and equivalently alleviates all of the associated symptoms and disabilities from low-grade spondylolisthesis and degenerative segmental instability. Furthermore, these patients seem to have optimal surgical indications for minimally invasive TLIF, while maintaining favorable surgical out comes.
\end{abstract}

Key Words: Spondylolisthesis, Transforaminal lumbar interbody fusion, Minimally invasive, Surgical outcome, Optimal indication

\section{Introduction}

Several studies have reported the favorable outcomes of minimally invasive transforaminal lumbar interbody fusion
(TLIF) performed with multiple surgical indications: lowgrade spondylolytic or degenerative spondylolisthesis, degenerative segmental instability, degenerative disc disease, post-laminectomy instability and spinal trauma [1-10]. However, each surgical indication could have its own

Received Feb 24, 2011; 1st Revised May 1, 2011; Accepted May 3, 2011

Corresponding author: Yung Park, MD

Department of Orthopedic Surgery, National Health Insurance Corporation Ilsan Hospital,

1232 Baekseok-dong, Ilsan-gu, Goyang 410-719, Korea

Tel: +82-31-900-0270, Fax:+ 82-31-900-0343, E-mail: yungspine@ gmail.com 
demographics and pain pattern and its own prognosis. If the outcome of a surgical procedure is evaluated according to multiple indications, then it is not clear how the procedure helped each subgroup of patients. Some indications could have better outcomes than the others, and so subgroup analyses should be performed with validated outcome measures to demonstrate the optimal surgical indications and treatment results of the procedure.

To the best of our knowledge, there has been no study that has evaluated the surgical indications and outcomes by subgroup analyses after minimally invasive TLIF. We have performed minimally invasive TLIF procedures in patients who were suffering from 3 major surgical indications, and these are spondylolytic spondylolisthesis, degenerative spondylolisthesis and degenerative segmental instability $[11,12]$. Our hypothesis is that minimally invasive TLIF optimally and equivalently alleviates the related symptoms and disabilities from these indications. The purpose of the study was to analyze the surgical outcomes of patients who underwent minimally invasive TLIF for the treatment of spondylolisthesis and degenerative segmental instability, and to determine whether these patients had optimal indications for minimally invasive TLIF.

\section{Materials and Methods}

\section{Patient's population}

We conducted a retrospective subgroup analysis of the prospectively collecting data from the consecutive patients who underwent single-level minimally invasive TLIF by a single surgeon at an institution for the treatment of the lowback pain and radiating pain down to the lower extremity (leg pain) associated with the following 3 subgroups: 1) adult acquired low-grade (grade I/II) spondylolytic spondylolisthesis, 2) grade I/II degenerative spondylolisthesis with segmental instability, and 3) degenerative segmental instability combined with lumbar stenosis (central, lateral and foraminal stenosis) and/or lumbar disc herniation. The inclusion criteria for segmental instability was $\geq 4 \mathrm{~mm}$ of translation or $\geq 10^{\circ}$ of angular motion seen on the preoperative flexion and extension radiographs.

Our relative contraindications for the minimally invasive approach (and the patients with these contraindications were treated by traditional open surgery) included 1) the patients with high-grade (grade III/IV) spondylolisthesis, 2) the patients with a severely collapsed disc space as well as no motion seen on the flexion-extension radiographs, 3) the patients who needed multi-level decompression and fusion, 4) the patients with combined coronal and/or sagittal deformities (kyphoscoliosis) that needed a correction, and 5) the patients who had back disease involving trauma, infection or pathologic causes.

All the patients underwent preoperative evaluation with static and dynamic plain lumbar radiographs, magnetic resonance imaging (MRI) and/or computed tomography (CT). Surgery was indicated if their preoperative signs and symptoms of low-back pain and leg pain were refractory to nonoperative treatment such as medications, physiotherapy and epidural steroid injection, or neurological deterioration developed. The surgical goal of all the patients was decompression and fusion for the treatment of the back pain and leg pain arising from lumbar segmental instability.

A total of 76 patients fulfilled the inclusion criteria of our study. Two patients died during the period of follow-up of causes not related to their index surgery, and 8 declined to participate the radiographic and $\mathrm{CT}$ evaluation at the final follow-up. Finally, 66 patients (87\%) completed the followup visit and were included in the analysis. There were 20 (30.3\%) men and 46 women with an average age of $57.5 \pm$ 9.2 years. The average duration of follow up was $36.1 \pm$ 9.9 months (range, 24 to 63 months). Thirteen patients (19.7\%) were current smokers. The coexisting conditions included 24 patients (36.4\%) with hypertension, $10(15.2 \%)$ patients with diabetes, and 16 (24.2\%) patients with osteoporosis. There were 3 subgroups and these included 23 patients with spondylolytic spondylolisthesis (group 1), 24 patients with degenerative spondylolisthesis (group 2) and 19 patients with degenerative segmental instabilities (group $3)$. The most common treated segment was L4-5 (40/66, 66.7\%), followed by L5-S1 (21/66) and then L3-4 (5/66). There were no conversions to an open procedure.

\section{Surgical technique}

Under fluoroscopic guidance, an appropriate-length 22 mm diameter METRx ${ }^{\mathrm{TM}}$ (Medtronic, Memphis, TN, USA) tubular retractor was introduced through a $2.5 \mathrm{~cm}$ incision for both neural decompression and access to the interbody space (Fig. 1). The approach was carried out on the side of the worst preoperative symptoms of radiculopathy. Sextant ${ }^{\text {TI }}$ (Medtronic) screws and rod were placed for percutaneous pedicle screw fixation (Fig. 2). A more detailed description of the procedure is available in the literature [1-10]. 


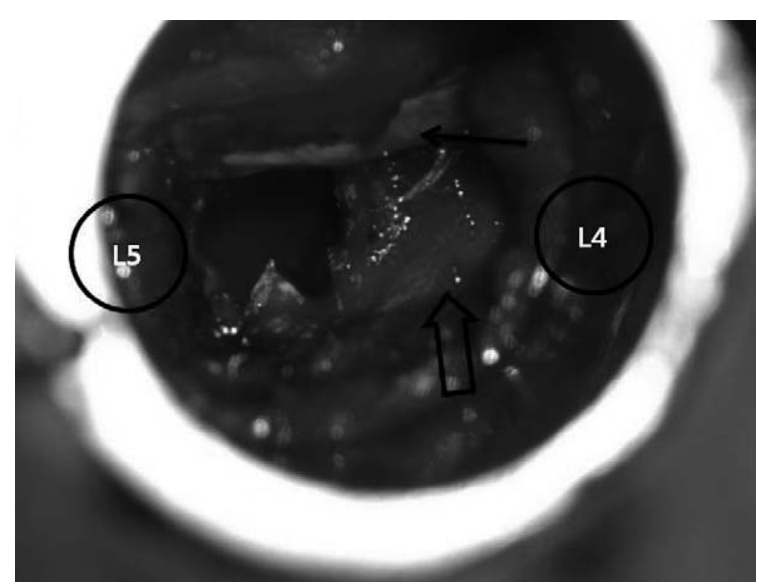

Fig. 1. Intraoperative microscopic view through a 22-mm tubular retractor demonstrating the right side transforaminal approach to L4-5. The inferior articular facet, the pars interarticularis and a portion of the lamina were removed. The locations of the L4 and L5 pedicles are defined by the black circles. The solid black arrow indicates the traversing root, and the open arrow points to the exiting root.
In all cases, the autogenous bone obtained from the resected lamina and facet and mixed with demineralized bone matrix (Osteofil ${ }^{\circledR}$ RT DBM paste, Regeneration Technologies Inc., Alachua, FL, USA) was placed anteriorly and contralateral to the annulotomy within the interbody space and then a polyether-ether-ketone (PEEK) cage (Capstone ${ }^{\circledR}$, Medtronic) was inserted into the disc space. No additional contralateral facet fusion was performed in all the patients.

\section{Clinical evaluation}

The data was analyzed from the prospectively collected complete medical records and the standardized questionnaires that were obtained at each visit. All the patients were evaluated pre- and post-operatively by one of the authors. The presence of symptoms, the use of pain medication, the functional status, the work status and the findings of a complete neurological examination were documented.
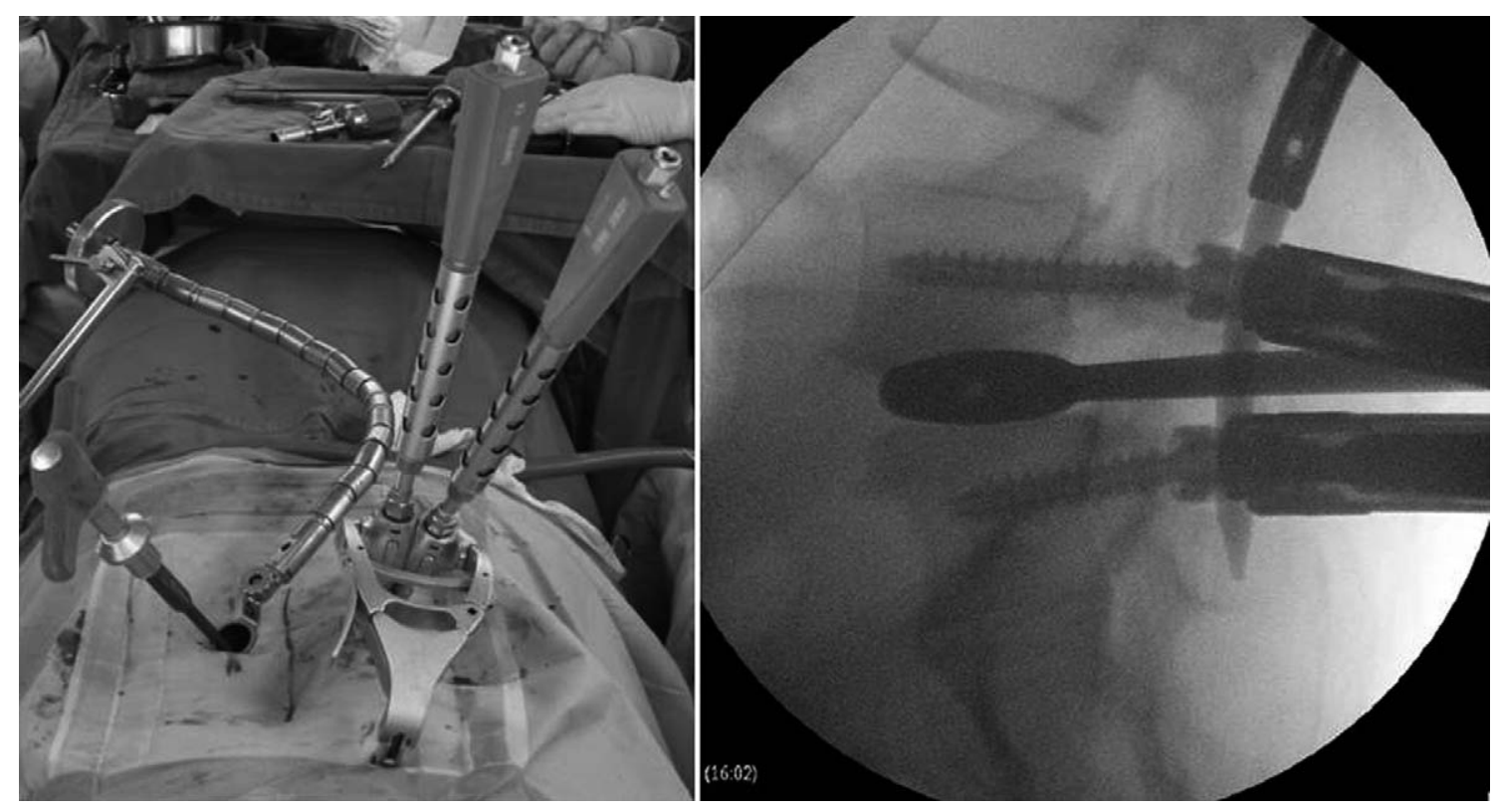

Fig. 2. Percutaneous pedicle screws and a rod were placed on the contralateral side to distract the disc space and then the screw-rod connections in the distracted position were provisionally tightened.

Table 1. Criteria for the assessment of functional outcome

\begin{tabular}{|c|c|c|c|c|}
\hline Outcome & Pain & Medication & Activity & Work status \\
\hline Excellent & None except for occasional back pain & None & Normal & Normal \\
\hline Good & Markedly improved, occational pain & $\begin{array}{l}\text { Occasional use of } \\
\text { pain medication }\end{array}$ & $\begin{array}{l}\text { Minimal functional } \\
\text { limitations }\end{array}$ & $\begin{array}{l}\text { Return to work, } \\
\text { although not at the same job activity }\end{array}$ \\
\hline Fair & Some improvement & $\begin{array}{l}\text { Frequent use of } \\
\text { pain medication }\end{array}$ & Restricted & Limited \\
\hline Poor & $\begin{array}{l}\text { No change in symptoms or a } \\
\text { worsening of the patient's condition }\end{array}$ & $\begin{array}{l}\text { Oral use of } \\
\text { narcotics }\end{array}$ & Incapacitated & Disabled \\
\hline
\end{tabular}


The clinical outcome was examined using the patientassessed quantitative measurement of the Visual Analogue Scale (VAS) for back pain/leg pain and the Oswestry Disability Index (ODI), as well as the surgeon-assessed outcome measurement using a modified functional scale (Table 1) [13], both preoperatively and at each postoperative follow-up. The VAS scores were recorded on a 10-mm horizontal line with 0 equal to "no pain," and 10 equal to "very severe pain." The ODI was scored on a 0-100 scale using the Oswestry Disability Questionnaire. The subjective postoperative symptoms documented at each postoperative visit were divided into 4 categories: symptom free, back pain only, leg pain only and both back and leg pain.

\section{Radiographic evaluation of fusion}

The standing anteroposterior, lateral, flexion and extension radiographs of the lumbosacral spine from the preoperative and final postoperative visits were reviewed for assessing the fusion. The postoperative CT scan was also obtained and evaluated at the final visit. The radiographic assessment of solid fusion was performed by 2 independent, experienced spine surgeons who were not involved with the surgical procedures.

A radiographic solid fusion was determined by the following criteria that were proposed by Burkus et al. [14]; 1) no motion (the acceptable intraobserver measurement error was $3^{\circ}$ angular motion or $3 \mathrm{~mm}$ of translation) on the flexion-extension lateral radiographs, 2) a continuous bony bridge within/around the cage (incorporation of the grafted bone into the vertebral endplates) was seen on the CT scan (Fig. 3A), 3) a lack of radiolucent lines around the graft and cage as well as the absence of a lucent hollow around the pedicle screws on the dynamic radiographs and/or CT scan, and 4) new bone formation adjacent to or within the cage (Fig. 3B) and/or fused posterior facet joint (the side opposite the TLIF approach) (Fig. 3C) on the CT scan.

A failure of fusion (nonunion) was considered according to the following categories: 1) changes in the sagittal-plane contours seen on the flexion-extension lateral radiographs, 2) the appearance of radiolucent lines at the cage-endplate interface and/or lucent hollows around the pedicle screw, and 3) cystic or sclerotic changes within the subchondral bone of the vertebral endplates seen on the plain radiographs and CT scan.

\section{Evaluation of complications}

The complications were subdivided into the intra- and post-operative complications: 1) the intra-operative complications included dura tear, hematoma, misplaced screw and cage migration and 2) the post-operative complications included infection, neurologic deficits, failure of instrumentation, failure of radiographic fusion and adjacent segment disease. Complications that were not specifically related to spine surgery and did not affect recovery (for example, urinary tract infection, ileus and anemia) were excluded.

Postoperative wound infection was defined as a deep infection requiring additional surgery such as debridement. Postoperative motor deficit was identified as a manual muscle strength test with a score $\leq 4$ on a scale of 0 to 5 . Adjacent segment disease was designated as a condition that required additional surgery for the treatment of symptoms refractory to non-operative treatment such as medication,
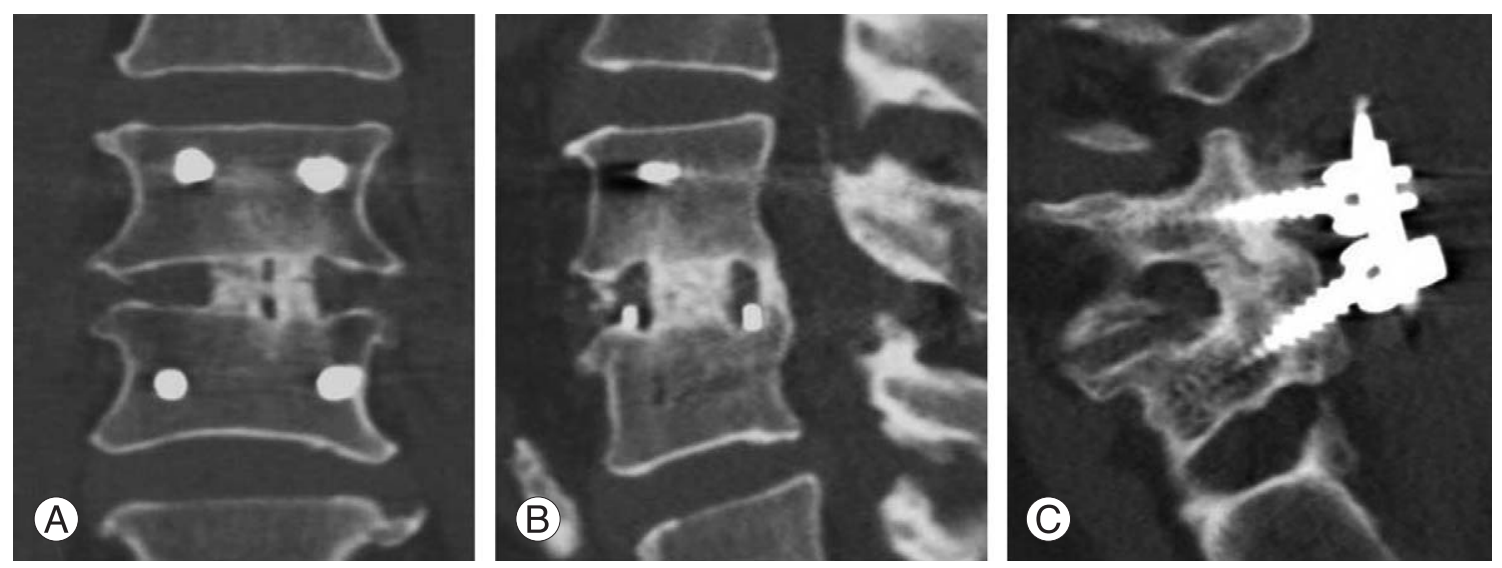

Fig. 3. Solid fusion is radiographically demonstrated on the sagittal and coronal computed tomography images. (A) There is continuous bony incorporation within and around the cage, $(\mathbf{B})$ new bone formation at the posterior margin of the interbody space and $(\mathbf{C})$ a fused posterior facet joint. 
physiotherapy and epidural steroid injection, or neurological deterioration due to adjacent segment degeneration. The newly developed or progressed abnormal processes, as compared with the preoperative radiographic findings, at the cranial and caudal segments of the fusion level and these included segmental instability (including antero/retrolisthesis), a herniated nucleus pulposus and stenosis were defined as adjacent segment degeneration. When the patients revealed newly developed signs and symptoms related with adjacent segment degeneration during follow-up, these lesions were determined by comparing the pre- and post-operative MRI and/or CT myelography.

\section{Statistical analyses}

The patients were divided into 3 subgroups according to their preoperative diagnosis: group 1 , spondylolytic spondylolisthesis; group 2, degenerative spondylolisthesis; and group 3, degenerative segmental instability.

The demographic data and the clinical and radiographic outcomes were compared among the 3 subgroups. The comparisons of data among the subgroups were base on chi- square tests for the categorical variables and on analysis of variance (ANOVA) for the continuous variables. Paired $t$ tests or Wilcoxon signed rank tests were used to evaluate the clinical improvement after surgery by analyses of the changes over time of the VAS and ODI scores between the 3 subgroups.

For all the analyses, a $p$-value less than 0.05 was considered to be statistically significant. The analyses were performed with the use of SPSS ver. 10.0 (SPSS Inc., Chicago, IL, USA).

\section{Results}

There was no significant difference of the demographic data among the 3 compared subgroups, as is shown in Table 2. The preoperative back pain, radiating leg pain (VAS scores) and disability (ODI scores) were significantly improved at the final postoperative follow-up in all of the subgroups (all measurements: $p<0.0001$ ) (Table 3).

The 3 subgroups showed equivalent back pain, leg pain and disability preoperatively and at final follow-up. The improvement of back pain, leg pain and disability after

Table 2. Demographic data of the patients treated with minimally invasive transforaminal lumbar interbody fusion from 2004 to 2006

\begin{tabular}{|c|c|c|c|c|c|}
\hline Demographics and characteristics & Overall & $\begin{array}{c}\text { Spondylolytic } \\
\text { spondylolisthesis } \\
\text { (group 1) }\end{array}$ & $\begin{array}{c}\text { Degenerative } \\
\text { spondylolisthesis } \\
\text { (group 2) }\end{array}$ & $\begin{array}{c}\text { Degenerative } \\
\text { segmental Instability } \\
\text { (group 3) }\end{array}$ & $p$-value ${ }^{\text {a) }}$ \\
\hline No. of patients & 66 & 23 & 24 & 19 & \\
\hline Mean age (range, yr) & $57.5(40-81)$ & $56(43-74)$ & $57.7(40-73)$ & $59.4(43-81)$ & 0.48 \\
\hline Female gender & $46(69.7)$ & $19(82.6)$ & $16(66.7)$ & $11(57.9)$ & 0.21 \\
\hline Mean body mass index (range, $\mathrm{kg} / \mathrm{m}^{2}$ ) & $24.6(18.2-34.6)$ & $25.6(18.2-30.9)$ & $24.0(19.1-34.6)$ & $24(18.8-27.6)$ & 0.15 \\
\hline$\leq 25$ & $37(56.1)$ & $9(39.1)$ & $18(75)$ & $10(52.6)$ & \\
\hline$>25$ & $29(43.9)$ & $14(60.9)$ & $6(25)$ & $9(47.4)$ & \\
\hline Mean height (range, $\mathrm{cm}$ ) & $156.6(142-178)$ & $153.9(145-169)$ & $158.3(144-169)$ & $158.7(142-178)$ & 0.11 \\
\hline ASA class $1 / 2 / 3 / 4$ & $23 / 43 / 0 / 0$ & $10 / 13 / 0 / 0$ & $8 / 16 / 0 / 0$ & $5 / 14 / 0 / 0$ & \\
\hline Patients who were smokers & $13(19.7)$ & $3(13)$ & $5(20.8)$ & $5(26.3)$ & 0.55 \\
\hline Patients who had osteoporosis $(\mathrm{T} \leq-2.5)$ & $16(24.2)$ & $7(30.4)$ & $3(12.5)$ & $6(31.6)$ & 0.24 \\
\hline Mean duration of symptoms (range, yr) & $2(0.5-20$ & $2.5(0.5-20)$ & $2(0.5-10)$ & $1.5(0.5-3)$ & 0.47 \\
\hline Mean duration of follow-up (range, yr) & $36.1(24-63)$ & $35.1(24-63)$ & $35.8(24-54)$ & $37.5(24-53)$ & 0.73 \\
\hline \multicolumn{6}{|l|}{ Meyerding grade } \\
\hline Grade 1 & 34 & 12 & 22 & & \\
\hline Grade 2 & 13 & 11 & 2 & & \\
\hline \multicolumn{6}{|l|}{ Level of surgery } \\
\hline L3-4 & 5 & 0 & 4 & 1 & \\
\hline L4-5 & 40 & 8 & 19 & 13 & \\
\hline L5-S1 & 21 & 15 & 1 & 5 & \\
\hline
\end{tabular}

Values are presented as number of patients (\%).

ASA: American society of anesthesiologists.

a) The p-values are base on chi-square tests for categorical variables and on analysis of variance (ANOVA) for continuous variables. 
surgery was also comparable among the 3 subgroups according to the paired differences of the VAS and ODI scores $(p=0.28, p=0.58, p=0.22)$. The detailed overall and compared clinical results are illustrated in Table 3 .

Furthermore, the 3 subgroups revealed similar distributions of the final postoperative symptoms (symptom free or not) and the final functional outcomes (excellent + good vs. fair + poor). Twelve patients (52\%) in group 1, 13 patients
(54\%) in group 2 and 8 patients (42\%) in group 3 showed no symptoms at the final visit. Additionally, 20 patients (87\%), 21 patients (88\%) and 13 patients (68\%) in each subgroup, respectively, showed excellent or good results according to the final functional scale (Table 4).

Fifty one patients achieved a radiographic solid fusion and 15 cases failed to achieve radiographic fusion. The overall rate of radiographic solid fusion was $77.3 \%$ (51/66).

Table 3. Overall and compared clinical results of 3 subgroups following minimally invasive transforaminal lumbar interbody fusion

\begin{tabular}{|c|c|c|c|c|c|}
\hline & Overall & Group 1 & Group 2 & Group 3 & p-value ${ }^{a)}$ \\
\hline No. of patients & 66 & 23 & 24 & 19 & \\
\hline \multicolumn{6}{|l|}{ VAS for back pain ${ }^{\text {b) }}$} \\
\hline Preoperative & $6.2 \pm 1.9$ & $6.7 \pm 1.8$ & $5.9 \pm 1.6$ & $5.8 \pm 2.3$ & 0.22 \\
\hline Final follow-up & $2.6 \pm 2.1$ & $2.8 \pm 2.3$ & $2.1 \pm 1.9$ & $3.0 \pm 2.2$ & 0.34 \\
\hline Paired differences & $3.6 \pm 2.4$ & $4.0 \pm 2.5$ & $3.8 \pm 2.3$ & $2.8 \pm 2.2$ & 0.28 \\
\hline $95 \%$ confidence interval of the difference & $3.0-4.2$ & $2.9-5.1$ & $2.8-4.8$ & $1.8-3.9$ & \\
\hline$p$-value ${ }^{\text {c) }}$ & $<0.0001$ & $<0.0001$ & $<0.0001$ & $<0.0001$ & \\
\hline \multicolumn{6}{|l|}{ VAS for leg pain ${ }^{\text {b) }}$} \\
\hline Preoperative & $8.1 \pm 2.0$ & $8.0 \pm 2.5$ & $8.2 \pm 1.4$ & $8.2 \pm 2.1$ & 0.92 \\
\hline Final follow-up & $1.6 \pm 2.8$ & $1.2 \pm 2.6$ & $1.4 \pm 2.6$ & $2.3 \pm 3.3$ & 0.43 \\
\hline Paired differences & $6.5 \pm 3.1$ & $6.8 \pm 3.2$ & $6.8 \pm 3.1$ & $5.9 \pm 3.1$ & 0.58 \\
\hline $95 \%$ confidence interval of the difference & $5.8-7.3$ & $5.4-8.2$ & $5.5-8.1$ & 4.4-7.4 & \\
\hline$p$-value ${ }^{\text {c) }}$ & $<0.0001$ & $<0.0001$ & $<0.0001$ & $<0.0001$ & \\
\hline \multicolumn{6}{|l|}{ ODI score ${ }^{\text {b) }}$} \\
\hline Preoperative & $60.2 \pm 16.5$ & $64.2 \pm 15.8$ & $55.7 \pm 14.8$ & $61.0 \pm 18.8$ & 0.21 \\
\hline Final follow-up & $25.9 \pm 17.9$ & $24.3 \pm 17.9$ & $23.0 \pm 17.3$ & $31.6 \pm 18.2$ & 0.25 \\
\hline Paired differences & $34.3 \pm 20.2$ & $39.9 \pm 20.1$ & $32.7 \pm 20.3$ & $29.4 \pm 19.5$ & 0.22 \\
\hline $95 \%$ confidence interval of the difference & 29.3-39.2 & $31.2-48.6$ & $24.2-41.3$ & $20.0-38.8$ & \\
\hline$p$-value ${ }^{c)}$ & $<0.0001$ & $<0.0001$ & $<0.0001$ & $<0.0001$ & \\
\hline
\end{tabular}

VAS: Visual Analogue Scale, ODI: Oswestry Disability Index.

${ }^{a}$ The p-values are base on analysis of variance (ANOVA) for the analyses of differences among 3 subgroups, ${ }^{\text {b }}$ The values are given as the mean and the standard deviation, ${ }^{\mathrm{c}}$ The $\mathrm{p}$ values are base on paired t-tests or Wilcoxon signed rank tests for the analyses of changes over time.

Table 4. Functional results among 3 subgroups after minimally invasive transforaminal lumbar interbody fusion

\begin{tabular}{|c|c|c|c|c|c|}
\hline & Overall & Group 1 & Group 2 & Group 3 & $p$-value ${ }^{\mathrm{a}}$ \\
\hline No. of patients & 66 & 23 & 24 & 19 & \\
\hline \multicolumn{6}{|l|}{ Final postoperative symptoms } \\
\hline Symptom free & $33(50)$ & $12(52)$ & $13(54)$ & $8(42)$ & 0.71 \\
\hline Back pain only & $16(24)$ & $6(26)$ & $6(25)$ & $4(21)$ & \\
\hline Leg pain only & $4(6)$ & $2(9)$ & $1(4)$ & $1(5)$ & \\
\hline Both back and leg pain & $13(20)$ & $3(13)$ & $4(17)$ & $6(32)$ & \\
\hline \multicolumn{6}{|l|}{ Final functional scale } \\
\hline Excellent & $37(56)$ & $14(61)$ & $15(63)$ & $8(42)$ & 0.25 \\
\hline Good & $17(26)$ & $6(26)$ & $6(25)$ & $5(26)$ & \\
\hline Fair & $10(15)$ & $3(13)$ & $2(8)$ & $5(26)$ & \\
\hline Poor & $2(3)$ & 0 & $1(4)$ & $1(5)$ & \\
\hline
\end{tabular}

The values are given as the number of patients (\%).

a) The $p$-values are base on chi-square tests for the analyses of categorical variables. 
The more complete data about the radiographic fusion status is shown in Table 5. The group with degenerative lumbar instabilities showed a higher fusion rate $(89.5 \%)$ than the other groups with spondylolisthesis $73.9 \%$ in the spondylolytic spondylolisthesis group and $70.8 \%$ in the degenerative spondylolisthesis group, respectively). Yet the difference was not statistically significant $(p=0.35)$ in regard to the fusion status between the 3 subgroups.

There were no complications such as intra-operative dura tears, screw misplacement, cage migration, neurologic deficits, failure of instrumentation and surgically related death among the 3 subgroups.

However, revision surgery was required for 1 patient in group 2 who developed adjacent segment disease and for 2 patients (1 deep wound infection and 1 adjacent segment disease) in group 3 to correct the postoperative complications. Additionally, there were 6 patients with nonunion in group 1,7 with nonunion in group 2 and 2 with nonunion in group 3. None of patients underwent revision surgery to repair the nonunion. There were comparable rates of complications among the 3 subgroups $(p=0.66)$ (Table 6 ).

One patient required a subsequent wound debridement secondary to infection 2 weeks after the index operation. Two patients underwent revision surgery due to adjacent segment disease and they had had initial improvement of the preoperative symptoms after their index surgery, and then they experienced a gradual worsening of the clinical and neurologic signs and symptoms, which consisted of back and/or leg pain, motor weakness and sensory disturbance, and these signs and symptoms were refractory to non-operative treatments such as medications and epidural steroid injection. Before revision surgery, they showed poor results of the functional scale, and they had an improvement of the clinical and neurologic signs and symptoms after surgery. Both cases occurred at the caudal segment (L4-5, L5-S1, respectively) of the index surgery. One patient underwent decompression and instrumented posterolateral fusion that connected to the previously fused segment, and the other patient was decompressed without fusion, each at the 24 and 47 months, respectively, after the index surgery.

\section{Discussion}

Minimally invasive TLIF has been reported to be a successful treatment modality for the patients with low-grade spondylolytic spondylolisthesis and degenerative lumbar instabilities, including degenerative spondylolisthesis [110]. Holly et al. [3] described that mechanical low-back and radiating pain associated with single-level grade I or II spondylolisthesis is one of the best indications for minimally invasive TLIF. Park and Foley [6] reported overall favorable results, as validated with the VAS and ODI scores of

Table 5. Radiographic fusion status among 3 subgroups after minimally invasive transforaminal lumbar interbody fusion

\begin{tabular}{lccccc}
\hline \hline & Overall & Group 1 & Group 2 & Group 3 & $p$-value \\
\hline No. of patients & 66 & 23 & 24 & 19 & $17(89.5)$ \\
Solid fusion & $51(77.3)$ & $17(73.9)$ & $15(70.8)$ & $7(29.2)$ & $2(10.5)$ \\
Nonunion & $15(22.7)$ & $6(26.1)$ & 0.35 \\
\hline
\end{tabular}

The values are given as the number of patients $(\%)$.

${ }^{a}$ The $p$-values are base on chi-square tests for the analyses of categorical variables.

Table 6. Complications among 3 subgroups after minimally invasive transforaminal lumbar interbody fusion

\begin{tabular}{|c|c|c|c|c|c|}
\hline & Overall & Group 1 & Group 2 & Group 3 & $p$-value ${ }^{\text {a) }}$ \\
\hline No. of patients & 66 & 23 & 24 & 19 & \\
\hline Dura tear & 0 & 0 & 0 & 0 & \\
\hline Failed or Misplaced implants & 0 & 0 & 0 & 0 & \\
\hline Neurologic deficits & 0 & 0 & 0 & 0 & \\
\hline Deep wound infection & 1 & 0 & 0 & 1 & \\
\hline Adjacent segment disease & 2 & 0 & 1 & 1 & \\
\hline Nonunion & 15 & 6 & 7 & 2 & \\
\hline Total number of complications & 18 & 6 & 8 & 4 & 0.66 \\
\hline Complication rate $(\%)$ & 27.3 & 26.1 & 33.3 & 21.1 & \\
\hline
\end{tabular}

The values are given as the number of patients.

a) The $p$-values are base on chi-square tests for the analyses of categorical variables. 
40 patients with low-grade spondylolisthesis, including 30 degenerative type patients and 10 spondylolytic type patients after minimally invasive TLIF. All the patients had their fusions confirmed radiographically by $\mathrm{CT}$ scans at the 2-year follow-up. Additionally, Schwender et al. [10] also reported a $100 \%$ fusion rate and significantly improved outcomes in 49 patients (22 with spondylolisthesis) at 1 year postoperatively, with decreasing VAS and ODI scores. In another study, Scheufler et al. [9] compared 53 patients who were undergoing minimally invasive TLIF to a historical cohort of 67 patients who underwent mini-open TLIF. Thirty-four of the 53 patients were treated for degenerative spondylolisthesis. At the 16-month follow-up, intermediate clinical success was noted based on the Roland-Morris low back pain scores and the American Academy of Orthopedic Surgeons lumbar spine questionnaires.

However, these results were analyzed together with multiple surgical indications without examining the subgroups. Since each indication has its own characteristic clinical and prognostic features, the outcomes validated with multiple indications would be too unclear to represent the selected outcome of each indication. To clarify this issue, we conducted subgroup analyses of patients who suffered from 3 common diseases after they were treated with minimally invasive TLIF by a single surgeon at one institution.

The current study demonstrated that patients having lowback pain and radiating leg pain coming from single-level, low-grade, spondylolytic or degenerative spondylolisthesis or degenerative segmental instability can be expected to have a significant and equivalent improvement from baseline, regarding the symptoms and disabilities, after minimally invasive TLIF. These patients could be the optimal candidates for the procedure, while maintaining similar clinical outcomes and radiographic fusion and complication rates as compared with each other.

Furthermore, the present study used criteria that included consistent radiographic clues to define a solid fusion such as new bone formation seen on CT scana. Burkus et al. [14] suggested that new bone formation adjacent to or within the cage is the most reliable radiographic indication of a solid fusion. Additionally, they also proposed the other criteria that substantiated radiographic solid fusion: no significant motion at the instrumented spinal segment on the dynamic studies, incorporation of grafted bone into the vertebral endplates and the absence of progressive radiographic lucent lines around the cage.

In the previous studies $[1,2,6,9,10]$, almost a $100 \%$ fusion rate was reported based on the flexion-extension radiographs and/or the postoperative CT scan at 2-years after minimally TLIF. In particular, recombinant human bone morphogenetic protein-2 (rhBMP-2) soaked in a collagen sponge carrier was used as a graft material in these series. Direct comparison of our fusion rate with other fusion rates appears to be inadequate because of the different graft materials and methods of measurement. The application of rigorous fusion criteria and the nonuse of rhBMP-2 might explain our relatively low rate of fusion compared to that of the previous studies [1-10].

The most commonly encountered complication in the current study was nonunion. Fortunately, the patients with radiographic nonunion did not complain much of pain and disability, which made revision to repair the nonunion unnecessary. Reducing the iatrogenic soft-tissue damage by performing a minimally invasive procedure might be the contributing reason why the patients with failed fusion did not have much back pain and disability. In addition, there were no complications such as dura violation, misplaced screws, cage migration and neurologic deficits, which have been reported in the previous studies [1-10]. On the contrary, 3 revision cases were noted during follow-up because of 2 cases of adjacent segment diseases and 1 case of postoperative infection. The overall complications rate for each indication was comparable with each other.

The present study has some limitations. Even though the clinical and radiographic data was prospectively collected, the current study is retrospective in nature and the patients were not randomly selected. In addition, the cohort size of this study is not large enough to have a sufficient statistical power for drawing complete conclusions for some aspects. For example, as our cohort included only 3 revision cases due to complications (2 ASD and 1 postoperative infection), and so only limited conclusions can be drawn about the complication rate from the results of the present study.

Another potential limitation of the study is that our average period of follow-up was long enough to sufficiently validate the effect of minimally invasive lumbar fusion on the outcomes such as adjacent segment degeneration. Furthermore, given the rarity of minimally invasive spinal fusion surgeries performed at multiple levels, the data from multiple segmental lumbar fusions was not available for the assessment as well. Finally, our study did not examine the radiographic measurements to determine the postoperative sagittal alignment, the lumbar lordosis and correction of a slip and a collapsed disc space. 


\section{Conclusions}

Despite of these weaknesses, our hypothesis was affirmed by the results of this current study, that is, minimally invasive TLIF optimally and equivalently alleviates the related symptoms and disabilities from single-level, low-grade, spondylolytic or degenerative spondylolisthesis or degenerative segmental instability. As a result of this present study, these patients seem to have the optimal surgical indications for undergoing a minimally invasive TLIF procedure, and the 3 subgroups had favorable surgical outcomes as compared with each other.

\section{REFERENCES}

1. Dhall SS, Wang MY, Mummaneni PV. Clinical and radiographic comparison of mini-open transforaminal lumbar interbody fusion with open transforaminal lumbar interbody fusion in 42 patients with long-term follow-up. J Neurosurg Spine 2008;9:560-5.

2. Foley KT, Holly LT, Schwender JD. Minimally invasive lumbar fusion. Spine (Phila Pa 1976) 2003;28(15 Suppl): S26-35.

3. Holly LT, Schwender JD, Rouben DP, Foley KT. Minimally invasive transforaminal lumbar interbody fusion: indications, technique, and complications. Neurosurg Focus 2006;20:E6.

4. Isaacs RE, Podichetty VK, Santiago P, et al. Minimally invasive microendoscopy-assisted transforaminal lumbar interbody fusion with instrumentation. J Neurosurg Spine 2005;3:98-105.

5. Mummaneni PV, Rodts GE Jr. The mini-open transforaminal lumbar interbody fusion. Neurosurgery 2005;57(4
Suppl):256-61.

6. Park P, Foley KT. Minimally invasive transforaminal lumbar interbody fusion with reduction of spondylolisthesis: technique and outcomes after a minimum of 2 years' follow-up. Neurosurg Focus 2008;25:E16.

7. Park Y, Ha JW. Comparison of one-level posterior lumbar interbody fusion performed with a minimally invasive approach or a traditional open approach. Spine (Phila Pa 1976) 2007;32:537-43.

8. Peng CW, Yue WM, Poh SY, Yeo W, Tan SB. Clinical and radiological outcomes of minimally invasive versus open transforaminal lumbar interbody fusion. Spine (Phila Pa 1976) 2009;34:1385-9.

9. Scheufler KM, Dohmen H, Vougioukas VI. Percutaneous transforaminal lumbar interbody fusion for the treatment of degenerative lumbar instability. Neurosurgery 2007;60(4 Suppl 2):203-12.

10. Schwender JD, Holly LT, Rouben DP, Foley KT. Minimally invasive transforaminal lumbar interbody fusion (TLIF): technical feasibility and initial results. J Spinal Disord Tech 2005;18 Suppl:S1-6.

11. Morgan FP, King T. Primary instability of lumbar vertebrae as a common cause of low back pain. J Bone Joint Surg Br 1957;39:6-22.

12. Dupuis PR, Yong-Hing K, Cassidy JD, Kirkaldy-Willis WH. Radiologic diagnosis of degenerative lumbar spinal instability. Spine (Phila Pa 1976) 1985;10:262-76.

13. Whitecloud TS 3rd, Davis JM, Olive PM. Operative treatment of the degenerated segment adjacent to a lumbar fusion. Spine (Phila Pa 1976) 1994;19:531-6.

14. Burkus JK, Foley K, Haid RW, LeHuec JC. Surgical Interbody Research Group--radiographic assessment of interbody fusion devices: fusion criteria for anterior lumbar interbody surgery. Neurosurg Focus 2001;10:E11. 